\title{
The Usefulness of the Interpretation of Initial Plain CT Scan in Hospitalized Patients with Acute Pyelonephritis without Obstructive Uropathy
}

\author{
Takehiro Sejima ${ }^{1, *}$, Shuichi Morizane ${ }^{2}$, Katsuya Hikita ${ }^{2}$, Masashi Honda ${ }^{2}$, Atsushi Takenaka ${ }^{2}$ \\ ${ }^{1}$ Department of Urology, Matsue City Hospital, Matsue, Japan \\ ${ }^{2}$ Division of Urology, Department of Surgery, Tottori University Faculty of Medicine, Yonago, Japan \\ Email address: \\ sejimat@matsue-cityhospital.jp (T. Sejima), morizane@med.tottori-u.ac.jp (S. Morizane), k-hikita@med.tottori-u.ac.jp (K. Hikita), \\ honda@med.tottori-u.ac.jp (M. Honda), atake@med.tottori-u.ac.jp (A. Takenaka) \\ ${ }^{*}$ Corresponding author
}

\section{To cite this article:}

Takehiro Sejima, Shuichi Morizane, Katsuya Hikita, Masashi Honda, Atsushi Takenaka. The Usefulness of the Interpretation of Initial Plain CT Scan in Hospitalized Patients with Acute Pyelonephritis without Obstructive Uropathy. International Journal of Infectious Diseases and Therapy. Vol. 4, No. 1, 2019, pp. 7-12. doi: 10.11648/j.ijidt.20190401.12

Received: February 8, 2019; Accepted: April 9, 2019; Published: May 15, 2019

\begin{abstract}
To investigate the association between plain computed tomography (CT) findings and clinical characteristics and to characterize the potential factors affecting the disease outcome in hospitalized patients with acute pyelonephritis (APN) without obstructive uropathy, we reviewed our clinical database retrospectively. Five plain CT scan abnormal kidney findings of perirenal fat stranding, thickening of perirenal fasciae, kidney enlargement, dilation of renal pelvis, and abnormal density of renal parenchyma were elucidated in 70 patients with APN without obstructive uropathy who were hospitalized. The association between CT scan findings and clinical characteristics were analyzed. Logistic regression analysis was performed to characterize the potential factors that affect the period until the patient was afebrile using various clinical factors and five defined CT scan findings. Perirenal fat stranding and dilation of renal pelvis were significantly frequent in females and patients with diabetes mellitus. Serum inflammation markers were significantly deteriorated in patients with perirenal fat stranding, thickening of perirenal fasciae and kidney enlargement compared to patients with normal findings. An age-adjusted Charlson comorbidity index $\geq 5$ and low serum albumin values were significantly associated with a prolonged febrile period. In conclusion, although abnormal plain $\mathrm{CT}$ scan findings at diagnosis are associated with some clinical characteristics, the patient's intrinsic factors are more useful for predicting the disease outcome in hospitalized patients with APN without obstructive uropathy.
\end{abstract}

Keywords: Computed Tomography, Hospitalized Patient, Kidney, Pyelonephritis

\section{Introduction}

Acute pyelonephritis (APN) is traditionally characterized by bacterial or fungal invasion of the kidney, causing acute interstitial inflammation and tubular cell necrosis. The diagnosis is mainly based on a combination of typical clinical symptoms and laboratory findings. The classical presentation is an abrupt onset of chills, fever and unilateral or bilateral flank or costovertebral angle pain and/or tenderness. These symptoms are often accompanied by dysuria, increased urinary frequency and urgency. In the laboratory tests, the patient may have leukocytosis with a predominance of neutrophils. Urinalysis usually reveals numerous leukocytes, which are often in clumps, and bacterial rods or chains of cocci.

Computed tomography (CT) is particularly recommended in severely ill patients who have a high risk of complications with atypical symptoms or no response to antibiotic therapy within 72 hours [1]. However, initial CT scanning provides important information on the range of inflammation and other accompanying complications to help to establish a precise diagnosis. The use of a multiphase CT protocol (pre- and post-contrast) has been widespread in current practice in APN [2-5]. Recently, Taniguchi et al. suggested that unenhanced 
and excretory CT phases are not really necessary because CT assessment of APN and urolithiasis can be accurately performed using only the late nephrographic phase [6]. However, physicians often hesitate to utilize enhanced CT scanning for prompt diagnosis of APN because they are afraid of renal functional deterioration and side effects of contrast material, especially in elderly patients with comorbidities or a poor general condition in the real clinical world. Plain CT scanning is a secure diagnostic method that is easy to use in clinical emergencies. Despite the clinical efficacy of several studies utilizing enhanced CT scanning, there is no study focusing on evaluation of the usefulness of plain CT scan. Moreover, the association between plain CT findings and clinical factors, including disease severity, has been poorly elucidated in APN.

In this study, we investigated the association between plain CT findings and clinical characteristics, and we characterized the potential factors affecting the disease outcome in APN without obstructive uropathy patients who underwent therapy in the hospital.

A)

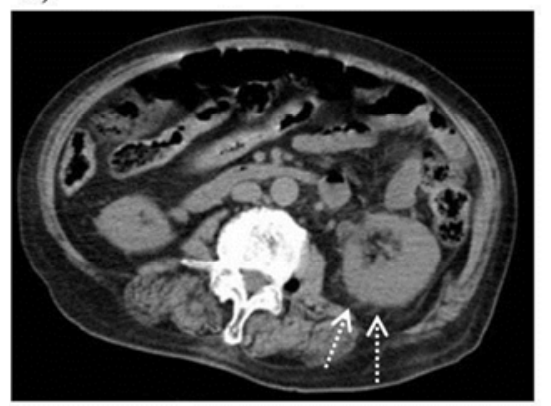

C)

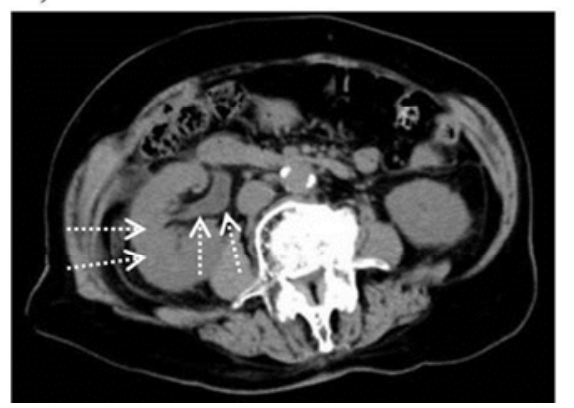

\section{Materials and Methods}

\subsection{Patients and the Definition of Abnormal CT Findings}

We retrospectively collected clinical data from 106 patients with APN without obstructive uropathy between January 2015 and December 2016. Of these patients, 70 who were admitted and underwent medical therapy were included in the study. The reason we focused on hospitalized patients is that all patients underwent plain CT scans and that the rate of abnormal findings in plain CT scans was relatively high because of the severity of APN without obstructive uropathy. The study was approved by an appropriately constituted ethics committee at our institution (Approval No. H30B-0012). The diagnostic definition of APN without obstructive uropathy was APN without any radiographic findings of obstructive uropathy. In terms of CT findings, five abnormal plain CT findings were defined (Figure 1). These findings are as follows: 1) perirenal fat stranding, 2) thickening of perirenal fasciae, 3) kidney enlargement, 4) dilation of renal pelvis, and 5) abnormal density of renal parenchyma.

B)

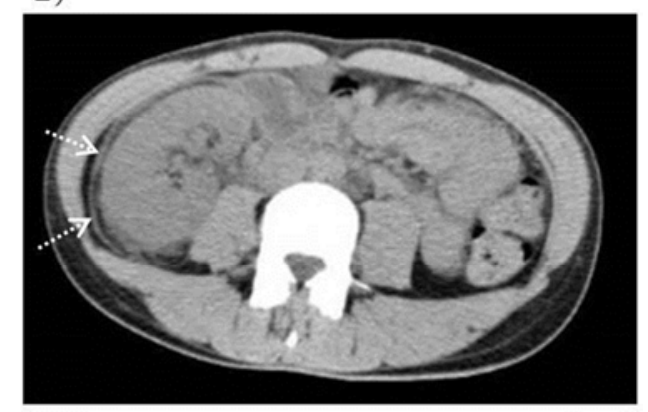

D)

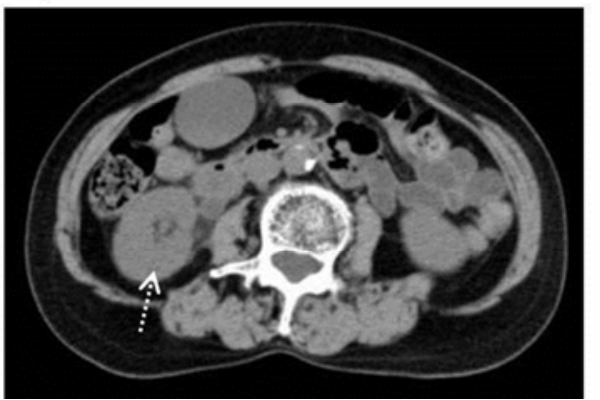

Figure 1. An example of abnormal plain computed tomography (CT) scan findings. Arrows indicate the findings. A) perirenal fat stranding, B) thickening of perirenal fasciae, C) kidney enlargement (facing right arrows) and dilation of renal pelvis (upward arrows), and D) abnormal density of renal parenchyma.

\subsection{The Analyses of the Association Between CT Findings and Clinical Factors}

All CT scan findings were retrospectively reviewed by a single radiologist (I. H.). The associations between five abnormal CT findings and clinical factors were analyzed. The analyzed clinical factors were categorized as nominal or continuous variables. The analyzed nominal factors are as follows: 1) gender (male vs. female), 2) diabetes mellitus (DM) (absence vs. presence), 3) past history of cardiovascular disease (CVD) (absence vs. presence), 4) age-adjusted Charlson comorbidity index (CCI) $(5>$ vs. $5 \leq)$,
5) The Eastern Cooperative Oncology Group performance status (ECOG PS) (0, 1 vs. 2 - 4), 6) disturbance of consciousness (absence vs. presence), 7) CVA pain (absence vs. presence), 8) systemic inflammatory response syndrome (SIRS) (absence vs. presence), 9) death due to APN (absence vs. presence), 10) white blood cell (WBC) reaction in urinalysis (negative vs. positive), 11) the number of WBCs in urinary sediment $(50>$ vs. $50 \leq), 12)$ blood bacterial culture (negative vs. positive), and 13) urine bacterial culture (negative vs. positive). Additionally, the analyzed continuous factors are as follows: 1) patient's age, 2) WBC count in peripheral blood, 3) platelet (PLT) count in 
peripheral blood, 4) serum C-reacted protein (CRP), 5) serum total protein (TP), and 6) serum albumin.

\subsection{The Analyses of the Potential Factors That Affect the Period Until the Patient Was Afebrile}

Because the most important evaluation of the effectiveness of medical therapy is whether the patient becomes afebrile, the duration from the date of therapy initiation to the date of afebrile status is critical. If the duration was over a week, the outcome of such cases should be considered deteriorated. Therefore, the potential factors that prolong the duration over seven days were searched using all analyzed clinical and radiological factors.

\subsection{Statistical Analyses}

The associations between abnormal CT finings and clinical nominal factors were analyzed using the chi-squared test. The associations between abnormal CT findings and clinical continuous factors were analyzed using Student's t test or Mann-Whitney test according to the fitness of the data to a Gaussian distribution. In searching the potential factors affecting the disease outcome of APN, logistic regression analysis was used.

\section{Results}

\subsection{The Baseline Patients' Clinical Characteristics and Outcome}

The data are summarized in table 1. Gender distribution was predominance of female, and most patients were elderly. General condition of patients was relatively poor. Namely, ECOG PS rated 2 and over and SIRS presentation at first visit were recognized in approximately half of patients. The median period until afebrile was five (range $0-30$ ). Three patients died due to the progression of pyelonephritis.

Table 1. Patient's clinical characteristics at first visit and outcome.

\begin{tabular}{llc}
\hline Characteristics & \multicolumn{2}{l}{ Values or Number of cases } \\
\hline \multirow{2}{*}{ Gender } & Male & 9 \\
Age (y) (mean, range) & Female & 61 \\
ECOG PS & $77.2(25-95)$ & \\
Age-adjusted CCI (median, range) & $0-1$ & 36 \\
& $2-4$ & 34 \\
Comorbidity & $4(0-7)$ & 24 \\
& No & 15 \\
Disturbance of consciousness & DM & 10 \\
CVA pain & Past history of CVD & 21 \\
Body temperature $\left({ }^{\circ} \mathrm{C}\right)$ (mean, range) & others & 63 \\
& No & 7 \\
Systolic blood pressure (mmHg) & Yes & 41 \\
& No & 29 \\
Heart rate (/min.) & Yes & \\
& $90>7(35.9-40.8)$ & 6 \\
& $90-160$ & 53 \\
& $160<$ & 11 \\
\end{tabular}

\begin{tabular}{llc}
\hline Characteristics & \multicolumn{2}{l}{ Values or Number of cases } \\
\hline \multirow{2}{*}{$\mathrm{SpO}_{2}(\%)$} & $92>$ & 17 \\
& $92-95$ & 30 \\
\multirow{2}{*}{$\mathrm{SIRS}$} & $95<$ & 23 \\
\multirow{2}{*}{ Period until afebrile (day) (median, range) } & No & 33 \\
\multirow{2}{*}{ Death due to APN } & $5(0-30)$ & 37 \\
& No & 67 \\
\hline
\end{tabular}

APN: acute pyelonephritis; CCI: Charlson comorbidity index; CVA: costovertebral angle; CVD: cardiovascular disease;

DM: diabetes mellitus; ECOG PS: The Eastern Cooperative Oncology Group performance status;

SIRS: systemic inflammatory response syndrome; $\mathrm{SpO}_{2}$ : arterial oxygen saturation of pulse oxymetry

\subsection{The Case Distributions According to Five Abnormal CT Findings}

The case distributions according to five abnormal CT findings are summarized in table 2. The common findings were perirenal fat stranding and thickening of perirenal fasciae, which were observed in 72.9 and $71.4 \%$ of cases, respectively. In contrast, an abnormal density of renal parenchyma was observed in only $10 \%$ of cases. Additionally, $74.3 \%$ of cases had more than two abnormal findings.

Table 2. Case distributions according to five abnormal CT findings.

\begin{tabular}{ll}
\hline CT finding & Number of cases (\%) \\
\hline Perirenal fat stranding & $19(27.1 \%)$ \\
No & $51(72.9 \%)$ \\
Yes & $20(28.6 \%)$ \\
Thickening of perirenal fasciae & $50(71.4 \%)$ \\
No & \\
Yes & $36(51.4 \%)$ \\
Kidney enlargement & $34(48.6 \%)$ \\
No & \\
Yes & $44(62.9 \%)$ \\
Dilation of renal pelvis & $26(37.1 \%)$ \\
No & \\
Yes & $63(90.0 \%)$ \\
Abnormal density of renal parenchyma \\
No & $7(10.0 \%)$ \\
Yes & \\
The number of abnormal CT findings & $6(8.6 \%)$ \\
0 & $12(17.1 \%)$ \\
1 & $52(74.3 \%)$ \\
$2 \leq$ &
\end{tabular}

CT: computed tomograph

\subsection{The Association Between Abnormal CT Finings and Clinical Factors}

In the analysis of nominal clinical factors, perirenal fat stranding and dilation of renal pelvis were significantly recognized in females and patients with DM, respectively. In the analysis of continuous clinical factors, some hematological parameters were significantly different between abnormal CT findings in negative and positive cases (Table 3). Regarding perirenal fat stranding, the values of CRP and PLT were significantly higher in positive cases than in negative cases. In contrast, the TP value was significantly 
lower in positive cases than in negative cases. With regard to thickening of perirenal fasciae, the TP value was significantly lower in positive cases than in negative cases. With regard to kidney enlargement, the CRP value was significantly higher in positive cases than in negative cases. With regard to the abnormal density of renal parenchyma, the PLT value was significantly higher in positive cases than in negative cases.

Table 3. The relationships between abnormal CT findings and continuous factors (age and hematological data).

\begin{tabular}{|c|c|c|c|c|c|}
\hline & Perirenal fat stranding & $\begin{array}{l}\text { Thickening of } \\
\text { perirenal fasciae }\end{array}$ & $\begin{array}{l}\text { Kidney } \\
\text { enlargement }\end{array}$ & $\begin{array}{l}\text { Dilation of renal } \\
\text { pelvis }\end{array}$ & $\begin{array}{l}\text { Abnormal density of } \\
\text { renal parenchyma }\end{array}$ \\
\hline & No / Yes & No / Yes & No / Yes & No / Yes & No / Yes \\
\hline Age & $75.3 / 78.9$ & $77.8 / 76.9$ & $76.8 / 78.4$ & $74.8 / 79.2$ & $76.9 / 77.6$ \\
\hline (mean; ys.) & $(P=0.256)$ & $(P=0.897)$ & $(P=0.452)$ & $(\mathrm{P}=0.187)$ & $(P=0.745)$ \\
\hline WBC & $13450 / 14520$ & $12710 / 14520$ & $14320 / 13690$ & $11910 / 15220$ & $13410 / 14780$ \\
\hline$($ mean; $/ \mu \mathrm{L})$ & $(\mathrm{P}=0.452)$ & $(P=0.256)$ & $(\mathrm{P}=0.892)$ & $(\mathrm{P}=0.092)$ & $(P=0.244)$ \\
\hline PLT & $13.4 / 18.1$ & $19.2 / 21.5$ & $18.2 / 23.3$ & $19.2 / 20.9$ & $17.0 / 24.1$ \\
\hline$\left(\right.$ mean; $\left.\times 10^{4} / \mu \mathrm{L}\right)$ & $(\mathrm{P}=0.031)$ & $(\mathrm{P}=0.398)$ & $(\mathrm{P}=0.084)$ & $(\mathrm{P}=0.432)$ & $(\mathrm{P}=0.037)$ \\
\hline CRP & $6.5 / 11.9$ & $8.2 / 9.8$ & $7.0 / 13.5$ & $6.9 / 10.8$ & $8.0 / 9.7$ \\
\hline (mean; mg/dL) & $(\mathrm{P}=0.039)$ & $(\mathrm{P}=0.128)$ & $(\mathrm{P}=0.006)$ & $(\mathrm{P}=0.083)$ & $(\mathrm{P}=0.118)$ \\
\hline TP & $6.8 / 6.3$ & $7.0 / 6.3$ & $6.7 / 6.4$ & $6.6 / 6.4$ & $6.7 / 6.3$ \\
\hline (mean; g/dL) & $(P=0.043)$ & $(P=0.008)$ & $(\mathrm{P}=0.167)$ & $(\mathrm{P}=0.672)$ & $(P=0.210)$ \\
\hline Albumin & $3.9 / 3.3$ & $3.6 / 3.8$ & $3.9 / 3.2$ & $3.8 / 3.5$ & $4.0 / 3.2$ \\
\hline (mean; g/dL) & $(P=0.145)$ & $(P=0.870)$ & $(\mathrm{P}=0.076)$ & $(\mathrm{P}=0.124)$ & $(\mathrm{P}=0.110)$ \\
\hline
\end{tabular}

CRP: C-reacted protein; CT: computed tomography; PLT: platelet; TP: total protein

\subsection{The Potential Factors Affecting the APN Disease Outcome}

In the first step, the potential factors that prolong the duration from the initiation of therapy until the patient is afebrile over seven days were analyzed with univariate analysis of all clinical and radiological factors. The age-adjusted CCI $(5 \leq)$, ECOG PS $(2-4)$, and lower value of
$\mathrm{TP}$ and serum albumin significantly prolonged the duration until afebrile. In the second step, multivariate analysis was performed with the factors that had a $\mathrm{P}$ value below 0.1 in the univariate analysis. Age-adjusted CCI $(5 \leq)$ and a lower value of serum albumin were independent factors prolonging the duration until afebrile (Table 4).

Table 4. Potential factors that prolong the duration from the initiation of therapy until the patient was afebrile over seven days.

\begin{tabular}{|c|c|c|c|c|}
\hline \multirow{2}{*}{ Factors } & \multicolumn{2}{|l|}{ Univariate Odds ratio } & \multicolumn{2}{|l|}{ Multivariate Odds ratio } \\
\hline & $(95 \% \mathrm{CI})$ & $P$ value & $(95 \% \mathrm{CI})$ & P value \\
\hline DM (Yes) & $4.571(0.821-25.469)$ & 0.0828 & $0.118(0.003-5.225)$ & 0.2642 \\
\hline Age-adjusted CCI $(5 \leq)$ & $10.678(1.874-60.941)$ & 0.0056 & $7.126(1.288-50.44)$ & 0.0345 \\
\hline ECOG PS $(2-4)$ & $6.545(1.179-36.331)$ & 0.0317 & $5.561(0.302-102.41)$ & 0.2483 \\
\hline Serum CRP & $1.098(0.993-1.215)$ & 0.0689 & $1.165(0.943-1.483)$ & 0.1571 \\
\hline Serum TP & $0.183(0.043-0.768)$ & 0.0103 & $0.344(0.152-2.26)$ & 0.2025 \\
\hline Serum albumin & $0.046(0.005-0.434)$ & 0.0071 & $0.131(0.006-2.979)$ & 0.0412 \\
\hline
\end{tabular}

CCI: Charlson comorbidity index; CI: confidence interval; CRP: C-reacted protein; DM: diabetes mellitus;

ECOG PS: The Eastern Cooperative Oncology Group performance status; TP: total protein

\section{Discussion}

The population of Japan is aging rapidly likewise other civilized nations. Therefore, increasing number of elderly patients with APN without obstructive uropathy is a critical problem. These patients are often advancing severity. Similarly, our patient characteristics showed aging and severe general condition. In fact, approximately half of patients were managed as sepsis because they all presented SIRS at the first visit. On the other hand, most patients with APN without obstructive uropathy are managed by non-urologists and residents in regional medical treatment. Therefore, it is difficult to diagnose such severe pyelonephritis using ultrasound which is known as appropriate imaging diagnostic method for uncomplicated APN. In such situation, the importance of diagnostic method using CT scan is emphasized. Our study was focused on the management of elderly patients with severe APN without obstructive uropathy.

In this study, we defined five abnormal plain CT findings that can easily be evaluated in the radiographic diagnosis. Our study demonstrated that the most common CT findings were perirenal fat stranding and thickening of perirenal fasciae in $72.9 \%$ and $71.4 \%$ of the cases, respectively. Renal parenchymal inflammation causes perirenal tissue changes that are recognized as perirenal fat stranding and thickening of perirenal fasciae. Kim et al. investigated the relationship between "uncommon CT findings" and clinical aspects in APN; however, the most common CT finding was perirenal fat infiltration (55\% of cases) [7]. Our results and previous 
studies suggest that the perirenal tissue change detected by CT scanning is a common pathogenesis of APN. Although the patient cohort had APN without obstructive uropathy, which was defined as APN without retention of urine passage from the anatomical and the physiological aspects, dilation of renal pelvis was seen in $37.1 \%$ of cases. Deteriorated ureter peristalsis caused by inflammation, a well-known pathophysiology of APN might cause dilation of the pelvis.

In the analysis of the association between abnormal CT findings and clinical nominal factors, our results demonstrated a positive relationship between perirenal fat stranding and dilation of renal pelvis with female gender and DM, respectively. The disease rate of APN was four-to five-fold higher in female patients from a large US database [8]. Similarly, our patient cohort showed female predominance (87.1\% of cases). Therefore, the significant association between CT abnormal findings and female gender is not conclusive because of the small sample size with a low rate of male gender. With regard to DM, it has been demonstrated that APN is five times more frequent in patients with DM than in non-DM patients and could lead to complications in approximately $30 \%$ of cases $[9,10]$. The significant high incidence of perirenal fat stranding in DM patients with APN without obstructive uropathy in our study suggests severe extension of inflammation and indicates there are therapeutic difficulties.

In the analysis of the association between abnormal CT finings and clinical continuous factors, one to three hematological inflammation markers (CRP, PLT, and TP) were significantly deteriorated in cases with perirenal fat stranding, thickening of perirenal fasciae, kidney enlargement or abnormal density of renal parenchyma. Kim et al reported on predictive severity of APN using nine criteria of abnormal CT scan findings and clinical factors that were indicated by the retrospective analysis of 193 APN patients [11]. In their study, global renal enlargement and abscess were part of the inclusion criteria. Although these two findings were obtained by enhanced CT scanning, it was possible that our findings of kidney enlargement and an abnormal renal parenchyma density correspond to global renal enlargement and abscess, respectively, in the previous study. In our four abnormal CT findings that were significantly associated with hematological inflammation markers, only perirenal fat stranding was associated with deterioration of all three markers (CRP, PLT, and TP). Therefore, perirenal fat stranding, which was a relatively frequent finding in our study, suggests the serious condition of APN.

All patients in our cohort were treated in the hospital. Pertel et al. reported on several risk factors, including hospitalization at baseline, for a poor outcome after therapy in outpatients and inpatients with uncomplicated APN [12]. Therefore, our patient cohort could be considered intractable to treatment. We emphasized the period until afebrile in such a characteristic patient cohort because afebrile status enables us to discharge patients from the hospital. Our study demonstrated that the age-adjusted CCI $(5 \leq)$ and a lower value of the serum albumin were independent factors affecting the prolonged duration until patients were afebrile. Although the serum albumin level is influenced by multifactorial factors, including inflammation progression of APN, these two independent factors are related to the host intrinsic features. Host intrinsic features, rather than direct inflammation, in APN were indicated as intractable factors for treatment in a previous study on an inpatient cohort in which prolonged hospitalization was noted among men and women with DM, long-term urinary catheterization, and a change in the initial antimicrobial therapy [13]. Careful observation and evaluation of patient characteristics in addition to hematological and radiographic inflammation findings at admission should be performed in APN.

The study has some limitations. First, the choice and method (intravenous or oral administration) of antibiotic agents depended on the policy decision of the physician in charge. A strict protocol for antibiotic therapy was not chosen. Therefore, it is possible that the period until the patients is afebrile might be affected by the skill of the physician in charge. However, this influence might be limited because the basic policy is that antibiotic agents should be selected based on the blood and urine bacterial culture results throughout the study period. Other limitations include the retrospective nature of the study and small sample size.

\section{Conclusion}

The assessment of APN without obstructive uropathy using initial plain CT scanning is a useful diagnostic method in patients who are treated in the hospital setting because of poor general condition, comorbidities and/or elderly age. Although abnormal plain CT scan findings at diagnosis are associated with clinical characteristics, the patient's intrinsic factors are more useful in predicting the disease outcome in APN without obstructive uropathy.

\section{Conflict of Interest Statement}

The authors declare that they have no competing interests.

\section{References}

[1] Stunell H, Buckley O, Feeney J, et al. Imaging of acute pyelonephritis in the adult. Eur Radiol. 2007; 17: 1820-1828.

[2] Lim SK, Ng FC. Acute pyelonephritis and renal abscesses in adults - correlating clinical parameters with radiological (computer tomography) severity. Ann Acad Med Singapore. 2011; 40: 407-413.

[3] Expert Panel on Urologic Imaging (2012) ACR Appropriateness Criteria Acute Pyelonephritis. Available at: https://acsearch.acr.org/docs/69489/Narrative/

[4] Oh SJ, Je BK, Lee SH, et al. Comparison of computed tomography findings between bacteremic and non-bacteremic acute pyelonephritis due to Escherichia coli. World J Radiol. 2016; 8: 403-409. 
[5] Yuh BI, Cohan RH, Francis IR, et al. Comparison of nephrographic with excretory phase helical computed tomography for detecting and characterizing renal masses. Can Assoc Radiol J. 2000; 51: 170-176.

[6] Taniguchi LS, Torres US, Souza SM, et al. Are the unenhanced and excretory CT phases necessary for the evaluation of acute pyelonephritis? Acta Radiol. 2017; 58: 634-640.

[7] Kim JS, Lee $\mathrm{S}$, Lee $\mathrm{KW}$, et al. Relationship between uncommon computed tomography findings and clinical aspects in patients with acute pyelonephritis. Korean J Urol. 2014; 55: 482-486.

[8] Ramakrishnan K, Scheid DC. Diagnosis and management of acute pyelonephritis in adults. Am Fam Physician. 2005; 71: 933-942.

[9] Tseng CC, Wu JJ, Wang MC, et al. Host and bacterial virulence factors predisposing to emphysematous pyelonephritis. Am J Kidney Dis. 2005; 46: 432-439.
[10] Scholes D, Hooton TM, Roberts PL, et al. Risk factors associated with acute pyelonephritis in healthy women. Ann Intern Med. 2005; 142: 20-27.

[11] Kim SH, Kim YW, Lee HJ. Serious acute pyelonephritis: a predictive score for evaluation of deterioration of treatment based on clinical and radiologic findings using CT. Acta Radiol. 2012; 53: 233-238.

[12] Pertel PE, Haverstock D. Risk factors for a poor outcome after therapy for acute pyelonephritis. BJU Int. 2006; 98: 141-147.

[13] Efstathiou SP, Pefanis AV, Tsioulos DI, et al. Acute pyelonephritis in adults: prediction of mortality and failure of treatment. Arch Intern Med. 2003; 163: 1206-1212. 